\title{
Theory of Microwave Parametric Down Conversion and Squeezing Using Circuit QED
}

\author{
K. Moon ${ }^{1,2}$ and S. M. Girvin ${ }^{1}$ \\ ${ }^{1}$ Sloane Physics Laboratory, PO Box 208120, \\ Yale University, New Haven, CT 06520-8120 \\ ${ }^{2}$ Department of Physics and Institute of Physics and Applied Physics, \\ Yonsei University, Seoul 120-749, Korea
}

(Dated: November 18, 2018)

\begin{abstract}
We study theoretically the parametric down conversion and squeezing of microwaves using cavity quantum electrodynamics of a superconducting Cooper pair box (CPB) qubit located inside a transmission line resonator. The non-linear susceptibility $\chi_{2}$ describing three-wave mixing can be tuned by dc gate voltage applied to the CPB and vanishes by symmetry at the charge degeneracy point. We show that the coherent coupling of different cavity modes through the qubit can generate a squeezed state. Based on parameters realized in recent successful circuit QED experiments, squeezing of $95 \% \sim 13 \mathrm{~dB}$ below the vacuum noise level should be readily achievable.
\end{abstract}

PACS numbers: 03.67.Lx, 74.50.+r, 32.80.-t, 42.50.Pq 
Squeezed states are a valuable tool to usefully manipulate the Heisenberg uncertainty principle by reducing the quantum fluctuations of a certain variable of interest at the expense of increased uncertainty for its conjugate variable. Using squeezed states one can perform very quiet measurements much below the vacuum noise level [1]. Squeezed states manifest the quantum coherent nature of light and provide a chance to beat the standard quantum limit by preferentially doing an experiment using the squeezed quadrature alone [2, 3]. Squeezed states have been experimentally observed in a nonlinear optical cavity experiment [4, 5]. Recently, the theory of squeezing in a high- $Q$ cavity was considered [6]. Upon the injection of a high-energy photon, a nonlinear optical medium can coherently generate two photons, the sum of whose frequencies is equal to that of the high-energy photon via optical parametric down conversion (PDC). If one injects low-energy photons instead, one may induce second harmonic generation, which also forms a squeezed state. In addition to this three-wave mixing, four-wave mixing can be used to generate squeezed states. In pioneering condensed matter experiments, the Josephson junction parametric amplifier was used in the microwave regime to produce $(47 \pm 8) \% \sim 3 \mathrm{~dB}$ squeezing below the vacuum level $[$,,$[8,[9]$ via degenerate four wave mixing. There has been tremendous recent progress in realizing quantum optics physics in electrical circuits. It is now possible to experimentally reach the extreme strong coupling limit of cavity QED [10, 11, 12] and to see very strong microwave non-linearities in high inductance small scale Josephson junctions whose Hamiltonian can be controlled with remarkable accuracy [13]. Coherent dynamics of a flux qubit coupled to a harmonic oscillator in a SQUID circuit has also been demonstrated [14].

Motivated by these experimental advances in 'circuit QED', we here study squeezing in a system consisting of a Cooper pair box (CPB) located inside a high $Q$ coplanar waveguide resonator. Two different discrete photon modes (fundamental and first harmonic) are coupled through the CPB as shown in Fig. 1 and squeezing occurs via three-wave mixing. Compared to the cavity QED of atomic physics, circuit QED has the advantages of infinite transit time of the 'atom' (qubit) inside the cavity and much stronger coupling between qubit and photon. We emphasize that strong coupling means we only need a single 'atom'. The circuit QED system recently successfully demonstrated strong coupling (vacuum Rabi splitting) between a single photon and a qubit in all solid state system [11]. While this architecture offers a very quiet environment leading to excellent coherence of the qubit $\left(T_{1} \sim 7 \mu \mathrm{s}\right.$, $\left.T_{2}^{*} \sim 500 \mathrm{~ns}\right)[12$, the solid-state environment still leads to qubit decay rates and dephasing 
rates much larger than those in corresponding atomic physics microwave cavity QED experiments [15]. Hence it is crucial to investigate these environmental effects on the efficiency of squeezing. Using both numerical and analytical calculations based on the currently available experimental parameters, we have estimated that the circuit QED system can readily produce about $95 \%$ squeezing, that is, $13 \mathrm{~dB}$ below the vacuum noise level.

We start with the following Hamiltonian to describe the coupled system of qubit and cavity photons for microwave circuit QED[10, 11]: $H=H_{0}+H_{I}$. The Hamiltonian $H_{0}$ is given by

$$
H_{0}=\frac{E_{01}}{2} \sigma_{z}+\hbar \omega_{1} a_{1}^{\dagger} a_{1}+\hbar \omega_{2} a_{2}^{\dagger} a_{2}
$$

where $E_{01}=\sqrt{E_{J}^{2}+E_{\mathrm{el}}^{2}}, E_{J}$ is the Josephson coupling energy, $E_{\mathrm{el}}=4 E_{C}\left(1-2 N_{g}\right), E_{c}$ the charging energy, $N_{g}$ the gate charge, and $\omega_{1}$ the angular frequency of the fundamental resonator mode and $\omega_{2}=2 \omega_{1}$ is the first harmonic frequency. The coupling Hamiltonian $H_{I}$ can be written

$$
H_{I}=-\left[g_{1}\left(a_{1}^{\dagger}+a_{1}\right)+g_{2}\left(a_{2}^{\dagger}+a_{2}\right)\right]\left[1-2 N_{g}+\sin \theta \sigma_{x}-\cos \theta \sigma_{z}\right],
$$

where $g_{i}$ represents the coupling strength between the qubit and the $i$ th cavity photon mode, and $\theta=\tan ^{-1}\left(E_{J} / E_{\mathrm{el}}\right)$. At the charge degeneracy point (CDP), that is, $N_{g}=1 / 2, H_{I}$ reduces to the Jaynes-Cummings Hamitonian, since $\cos \theta \cong-\left(4 E_{C} / E_{J}\right)\left(1-2 N_{g}\right)=0$ and $\sin \theta=1$. One can see that away from the CDP, we have couplings other than the JaynesCummings term, whose strength linearly increases with the deviation. Because the only relevant cavity frequencies will be the fundamental and first harmonic, we expect degenerate PDC to occur in our system, where a single high-energy photon coherently generates two photons, each with half the frequency. We emphasize that this is degenerate PDC because the cavity only has discrete modes. In order to achieve squeezing via PDC in circuit QED, the system should be able to convert a single $\omega_{2}$ photon into two $\omega_{1}$ photons, which requires a term of the form $a_{1}^{\dagger} a_{1}^{\dagger} a_{2}$ in the effective Hamiltonian. This will result from the third order processes in terms of $H_{I}$, which will be in general negligible since $g_{1}^{2} g_{2} / \omega_{1}^{3} \ll 1$ in typical experiments[11]. However we can resonantly enhance the process by tuning either $\omega_{1}$ or $\omega_{2}$ close to $E_{01}$. We have chosen the case $\omega_{2} \cong E_{01}$.

We first apply the following unitary transformation $U_{1}$ to the Hamiltonian $H$

$$
U_{1}=\exp \left[\sum_{i=1,2} \frac{g_{i}}{\omega_{i}}\left(a_{i}-a_{i}^{\dagger}\right)\left(1-2 N_{g}-\cos \theta \sigma_{z}\right)\right] \text {. }
$$


This corresponds to shifting the centers of the harmonic oscillator coordinates $X_{i}=a_{i}+a_{i}^{\dagger}$ by $\left(2 g_{i} / \omega_{i}\right)\left(1-2 N_{g}-\cos \theta \sigma_{z}\right)$. Subsequently, we apply the unitary transformation

$$
U_{2}=\exp \left[\frac{g_{1} \sin \theta}{2\left(E_{01}-\omega_{1}\right)}\left(a_{1}^{\dagger} \sigma^{-}-a_{1} \sigma^{+}\right)\right] .
$$

Upon application of these two unitary transformations, we obtain after perturbative expansion in $g_{1}$ and $g_{2}$, the following Hamiltonian: $H_{\text {eff }}=U_{2} U_{1} H U_{1}^{\dagger} U_{2}^{\dagger}=H_{0}+H^{\prime}$ for $E_{01} \cong \omega_{2}$,

$$
H^{\prime}=-\frac{1}{2} g_{2} \sin \theta\left(a_{2}^{\dagger} \sigma^{-}+a_{2} \sigma^{+}\right)+\frac{g_{1}^{2} \sin 2 \theta}{2 \omega_{1}}\left(a_{1}^{\dagger} a_{1}^{\dagger} \sigma^{-}+a_{1} a_{1} \sigma^{+}\right) .
$$

We define the energy detuning between the cavity photon frequency $\omega_{2}$ and the qubit energy splitting $E_{01}$ to be $\Delta \equiv E_{01}-\omega_{2}$ and consider the case $g_{2} \ll \Delta \ll \omega_{1}$. It is this Hamiltonian which we will study numerically. However to develop an analytical understanding, we can apply the following additional unitary transformation $U_{3}$

$$
U_{3}=\exp \left[\frac{g_{2} \sin \theta}{2 \Delta}\left(a_{2}^{\dagger} \sigma^{-}-a_{2} \sigma^{+}\right)\right] .
$$

Finally, we obtain the following low-energy effective Hamiltonian through $\hat{H}_{\text {eff }}=U_{3} H_{\text {eff }} U_{3}^{\dagger}$,

$$
\hat{H}_{\text {eff }}=H_{0}+\frac{g_{2}^{2} \sin ^{2} \theta}{2 \Delta}\left[1+\sigma_{z}\left(2 n_{2}+1\right)\right]+\frac{\zeta}{2}\left(a_{1}^{\dagger} a_{1}^{\dagger} a_{2}+a_{1} a_{1} a_{2}^{\dagger}\right) \sigma_{z},
$$

where the second term on the right represents the Lamb and light shifts of the qubit splitting frequency and $\zeta=\left(2 g_{1}^{2} g_{2} \sin \theta \sin 2 \theta / \Delta \omega_{1}\right)$. The third term is the desired squeezing term. $\hat{H}_{\text {eff }}$ is exactly the standard Hamiltonian for degenerate optical PDC including the Lamb shift [3]. Note that the squeezing operator couples to the qubit state $\sigma_{z}$ and the phase of the squeezed quadrature will shift by $\pi / 2$ if the qubit is placed in the excited state.

In deriving the above result, we have neglected the effect of cavity damping $\sum_{i=1,2} \sqrt{\kappa_{i}} \int_{-\infty}^{\infty} d \omega a_{i} b_{\omega}^{\dagger}$, where $b_{\omega}$ represents the continuum modes outside the cavity and $\kappa_{i}$ the cavity loss rates. Unitary transformation of the damping terms leads to radiative atom damping [10] and two-photon decay terms [16] such as $\left(\sqrt{\kappa_{2}} \zeta / 2 \Delta\right) \int_{-\infty}^{\infty} d \omega a_{1} a_{1} b_{\omega}^{\dagger}$. The two-photon decay rate is much smaller than the cavity loss $\kappa_{2}$ by a factor of $(\zeta / \Delta)^{2} / 2$, and hence we will neglect it. Similarly the radiative atom damping term is smaller than the intrinsic atom decay rate and we will neglect it. In the limit of weak pumping, down converted pairs of photons are produced incoherently at a rate $\bar{n}_{2}\left(\zeta / \kappa_{1}\right)^{2}$ given by Fermi's golden rule with $\bar{n}_{2}$ the average number of pump photons. 
To understand the squeezing produced by strong pumping, one needs to consider the substantial de-excitation rate $\gamma$ from the excited state of solid-state qubits. Furthermore, the qubit dephasing rate $\gamma_{\varphi}$ is typically at least one order of magnitude greater than $\gamma$. For charge qubits, optimal phase coherence occurs at the charge degeneracy point 17] where (it happens that) the symmetry prevents the three wave mixing which we require for PDC. Hence it is crucial to take into account these effects to obtain a realistic estimate of squeezing. For a deviation of $10 \%$ from the charge degeneracy point, coherence times of $T_{2} \sim 200 \mathrm{~ns}$ have been demonstrated [17]. We start with the Hamiltonian $\tilde{H}_{I}$ obtained via the unitary transformation $U_{1} U_{2}$ in Eq. 5. which is defined in the rotating wave frame of $\omega_{2}$,

$$
\tilde{H}_{I}=\frac{\Delta}{2} \sigma_{z}+g_{2} \sin \theta\left|\alpha_{p}\right| \sigma_{y}+\frac{\Gamma}{4}\left(a_{1}^{\dagger} a_{1}^{\dagger} \sigma^{-}+a_{1} a_{1} \sigma^{+}\right) .
$$

Here $\Gamma=2 g_{1}^{2} \sin 2 \theta / \omega_{1}$ and we have taken the pump to be classical: $\left\langle a_{2}\right\rangle=\alpha_{p}=i\left|\alpha_{p}\right|$.

Following the standard quantum theory of damping, we investigate the coupled system of qubit and cavity plus the reservoir. After integrating out the reservoir degrees of freedom and using the Markov approximation, one obtains the master equation [3] for the reduced density matrix $\rho$ of the qubit plus cavity system,

$\frac{d \rho}{d t}=-i\left[\tilde{H}_{I}, \rho\right]+\kappa_{1}\left[a_{1} \rho a_{1}^{\dagger}-\frac{1}{2} a_{1}^{\dagger} a_{1} \rho-\frac{1}{2} \rho a_{1}^{\dagger} a_{1}\right]+\frac{\gamma}{4}\left[\sigma^{-} \rho \sigma^{\dagger}-\frac{1}{2} \sigma^{\dagger} \sigma^{-} \rho-\frac{1}{2} \rho \sigma^{\dagger} \sigma^{-}\right]+\frac{\gamma_{\varphi}}{2}\left[\sigma_{z} \rho \sigma_{z}-\rho\right]$.

Using the quantum regression theorem [3], the variances $V(\omega)$ (homodyne spectrum) of quadrature $X_{1}=a+a^{\dagger}$ and $X_{2}=\left(a-a^{\dagger}\right) / i$ for the output cavity photon mode are given by

$$
V(\omega)=1 \pm \kappa_{1} \int_{-\infty}^{\infty} d \tau e^{-i \omega \tau} \operatorname{Tr}\left\{\left(a_{1} \pm a_{1}^{\dagger}\right) e^{\mathcal{L} \tau}\left(a_{1} \rho_{s s} \pm \rho_{s s} a_{1}^{\dagger}\right)\right\}
$$

where $\mathcal{L}$ is the Liouvillean operator, $\rho_{s s}$ the density matrix at the steady state, and the $(+)$ and $(-)$ sign correspond to $X_{1}$ and $X_{2}$ quadratures, respectively. We have numerically calculated the $V(\omega)$ of quadrature $X_{1}$ and $X_{2}$ based on an exact diagonalization study in the Hilbert space of size $2 N \times 2 N$ corresponding to the two possible spin states and the number of photons being restricted to less than $N$. We have chosen the following conservative set of experimental parameters: $\omega_{1} / 2 \pi=3 \mathrm{GHz}, T_{1}=2 \mu \mathrm{s}, T_{2}=100 \mathrm{~ns}, Q=$ $5 \times 10^{5}, g_{2} / 2 \pi=18 \mathrm{MHz}, E_{C} / E_{J} \cong 1$, and $\sin \theta \cong 1$ near the CDP, where $T_{2}=\gamma_{\perp}^{-1}$, $\gamma_{\perp}=\gamma_{\varphi}+\gamma / 2$, and $\Delta / \omega_{1} \cong 0.05[11]$. In Fig. 2] we have plotted $V(\omega)$ as a function of $\omega$ for $\Delta=2.6 \times 10^{4}, \Gamma=5, N=10$, and $g_{2}\left|\alpha_{p}\right|=2827$ in units of $\kappa_{1}=\omega_{1} / Q$. We find that the maximum squeezing is obtained at $\omega=0$ as expected. The frequency width of 
the squeezing spectrum is controlled by the cavity width $\kappa_{1}$. The variable $\Gamma$ is tunable by varying the qubit gate voltage. It is experimentally observed that the dephasing time $T_{2}$ decreases very rapidly, as the qubit is detuned from the CDP [17]. Hence we will restrict the relative deviations from the CDP to be small $\left|2 N_{g}-1\right| \leq 0.1$, where $T_{2} \geq 100 \mathrm{~ns}$. This restriction yields a maximum value of $\Gamma \cong\left(8 g_{2}^{2} / \omega_{1}\right)\left(E_{C} / E_{J}\right)\left(1-2 N_{g}\right) \sim 0.53 \mathrm{MHz}$. In Fig. [3. $V(\omega=0)$ is plotted as a function $\Gamma$ for $N=10,12,15,20$. We have checked that $\Delta X_{1} \cdot \Delta X_{2}$ satisfies the minimum uncertainty bounds and closely approaches the minimum uncertainty condition. The maximum output squeezing is obtained by extrapolation of the numerical results for finite $N$ to $N=\infty$ yielding $V(\omega=0)=0.05$, that is, $-13 \mathrm{~dB}$, as shown in the inset of Fig. 3 ,

We have performed extensive simulations by varying the sets of parameters. Increasing $\Delta$ consistently improves the squeezing because it reduces the effect of dephasing of the qubit. We can analytically study the effect of finite qubit decay time and dephasing on the squeezing as follows. Based on perturbative analysis, we have demonstrated that the PDC rate is given by $\left(g_{2} \Gamma \sin \theta / \Delta\right) \sigma_{z}$ as shown in Eq. 7. When concerned with the spin dynamics of the qubit alone, we may neglect the coupling term between cavity photon and the qubit, which is much smaller than the other terms in Eq. 8. By neglecting the coupling, we obtain the following steady state solution for the spin polarization $\left\langle\sigma_{z}\right\rangle:\left\langle\sigma_{z}\right\rangle=$ $-\gamma\left(\gamma_{\perp}^{2}+\Delta^{2}\right) /\left(4 g_{2}^{2}\left|\alpha_{p}\right|^{2} \sin ^{2} \theta \gamma_{\perp}+\gamma\left(\gamma_{\perp}^{2}+\Delta^{2}\right)\right)$. In the absence of pump photons, $\left(\left|\alpha_{p}\right|=0\right)$, the qubit correctly decays down to the ground state, that is $\left\langle\sigma_{z}\right\rangle=-1$. For large detuning, the finite pump only slightly quenches the spin polarization.

Due to the quenching of qubit spin, the effective PDC parameter $\chi_{2}$ is given by the following formula

$$
\chi_{2}=\frac{g_{2} \sin \theta\left|\alpha_{p}\right| \Gamma}{\Delta} \frac{\gamma_{\perp}^{2}+\Delta^{2}}{4 g_{2}^{2}\left|\alpha_{p}\right|^{2} \sin ^{2} \theta\left(\gamma_{\perp} / \gamma\right)+\gamma_{\perp}^{2}+\Delta^{2}} .
$$

Maximum squeezing is achieved near the critical point $\chi_{2} \cong \kappa_{1} / 2[3]$, which leads to the optimal value of $\Gamma$ for $\Delta \gg \gamma_{\perp}$

$$
\Gamma^{*}=\frac{\kappa_{1}}{2 x}\left[1+4\left(\gamma_{\perp} / \gamma\right) x^{2}\right]
$$

where $x=g_{2} \sin \theta\left|\alpha_{p}\right| / \Delta$. In Fig. 团 $\Gamma^{*}$ is plotted as a function of $\Delta$. The dotted line represents the result from the analytical formula of Eq. 12 which shows excellent agreement with the numerical simulation (filled circles). By differentiating with respect to $x$, one 
can obtain the minimum value of $\Gamma^{*}$ required to achieve the maximum squeezing: $\Gamma_{\min }^{*}=$ $2 \kappa_{1}\left(\gamma_{\perp} / \gamma\right)^{1 / 2}$ for $x^{*}=\left(\gamma / 4 \gamma_{\perp}\right)^{1 / 2}$. Since the value of the $\Gamma / \kappa_{1}$ can reach as large as 13.3 for the chosen set of parameters, it is well above $\Gamma_{\min }^{*} / \kappa_{1}=2\left(\gamma_{\perp} / \gamma\right)^{1 / 2} \cong 8.9$. Hence the maximum squeezing can be realized with the chosen experimental parameters. The validity of perturbation theory in $g_{2}$ [10] imposes the following constraint on the product of the pump amplitude and the coupling, $g_{2}\left|\alpha_{p}\right|<(\Delta / 2 \sin \theta)$. We note that one can control the values of $g_{1}$ and $g_{2}$ independently by shifting the position of the qubit within the cavity. When the CPB is located at $1 / 4$ of the resonator length from the left edge, $g_{2}$ vanishes as shown in Fig. 1] Hence by placing the CPB slightly left of the above position, the pump amplitude can always be made large enough for the classical approximation to be valid.

In summary, we have studied degenerate parametric down conversion and squeezing in circuit QED, where a superconducting Cooper pair box (CPB) qubit is located inside a transmission line resonator. We have shown that away from the charge degeneracy point, the coherent coupling of different cavity modes through the qubit can generate a squeezed state via three-wave mixing. We have investigated the effect of the finite qubit lifetime and dephasing on squeezing, which will be crucial especially for the qubit away from the charge degeneracy point. By performing both the numerical and analytical calculations, we have demonstrated that the squeezing of about $13 \mathrm{~dB}$ below the vacuum noise level can be obtained for the currently available experimental parameters.

We thank A. Blais and J. Gambetta for valuable discussions and guidance in the quantum optics simulations. This work was supported in part by the National Security Agency (NSA) and Advanced Research and Development Activity (ARDA) under Army Research Office (ARO) contract numbers DAAD19-02-1-0045 and ARDA-ARO W911NF-05-1-0365, and by NSF ITR-0325580, NSF DMR-0342157 and the Keck Foundation. K.M. wishes to acknowledge the financial support of the LG Yonam Foundation and the National Program for Tera-Level Nanodevices of the Korea Ministry of Science and Technology as one of the 21 Century Frontier Programs.

[1] Q. A. Turchette, N. Ph. Georgiades, C. J. Hood, and H. J. Kimble, and A. S. Parkins, Phys. Rev. A 58, 4056 (1998). 
[2] P.D. Drummond, in Quantum Squeezing, edited by P.D. Drummond and Z. Ficek (Springer, 2003) pp. 100-139.

[3] D. F. Walls and G. J. Milburn, Quantum Optics, (Springer, 1994).

[4] R. E. Slusher, L. W. Hollberg, B. Yurke, and J. C. Mertz, Phys. Rev. Lett. 55, 2409 (1985).

[5] L. A. Wu, H. J. Kimble, J. L. Hall, and H. Wu, Phys. Rev. Lett. 57, 2520 (1986).

[6] N. G. de Almeida, R. M. Serra, C. J. Villas-Boas, and M. H. Y. Moussa, Phys. Rev. A 69, 035802 (2004) and references therein.

[7] B. Yurke, J. Opt. Soc. Am. B, 4, 1551 (1987).

[8] B. Yurke, P. G. Kaminsky, R. E. Miller, E. A. Whittaker, A. D. Smith, A. H. Silver, and R. W. Simon, Phys. Rev. Lett. 60, 764 (1988).

[9] R. Movshovich, B. Yurke, P. G. Kaminsky, A. D. Smith, A. H. Silver, and R. W. Simon, and M. V. Schneider, Phys. Rev. Lett. 65, 1419 (1990).

[10] A. Blais, R.S. Huang, A. Wallraff, S. M. Girvin, and R.J. Schoelkopf, Phys. Rev. A 69, 062320 (2004).

[11] A. Wallraff, D. I. Schuster, A. Blais, L. Frunzio, R.-S. Huang, J. Majer, S. Kumar, S. M. Girvin, R. J. Schoelkopf, Nature (London) 431, 162-167 (2004).

[12] D. I. Schuster, A. Wallraff, A. Blais, L. Frunzio, R.-S. Huang, J. Majer, S. M. Girvin, R. J. Schoelkopf, Phys. Rev. Lett. 94, 123602 (2005).

[13] I. Siddiqi, R. Vijay, F. Pierre, C. M. Wilson, M. Metcalfe, C. Rigetti, L. Frunzio, and M. H. Devoret Phys. Rev. Lett. 93, 207002 (2004); I. Siddiqi, R. Vijay, F. Pierre, C. M. Wilson, L. Frunzio, M. Metcalfe, C. Rigetti, R. J. Schoelkopf, M. H. Devoret, D. Vion, and D. Esteve Phys. Rev. Lett. 94, 027005 (2005).

[14] I. Chiorescu, P. Bertet, K. Semba, Y. Nakamura, C. J. P. M. Harmans, J. E. Mooij, Nature (London) 431, 159-162 (2004).

[15] J. Raimond, M. Brune, and S. Haroche, Rev. Mod. Phys. 73, 565 (2001).

[16] B. Yurke and E. Buks, quant-ph/0505018 (2005).

[17] D. Vion, A. Aassime, A. Cottet, P. Joyez, H. Pothier, C. Urbina, D. Esteve, M. H. Devoret, Science 296, 886-889; Fortschr. Phys. 51, 462 (2003). 


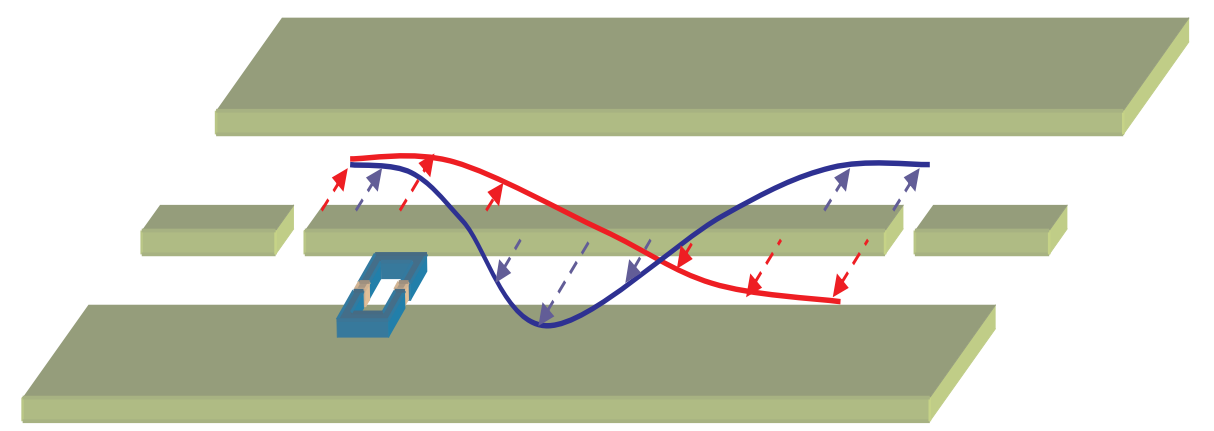

FIG. 1: (Color online) The schematic diagram for the circuit QED coupled to a Cooper-pair box located at one edge of the cavity. The red and blue lines represent the fundamental and the second harmonic cavity modes, respectively. The change of CPB position from the edge will vary the coupling strengths $g_{1}$ and $g_{2}$. 


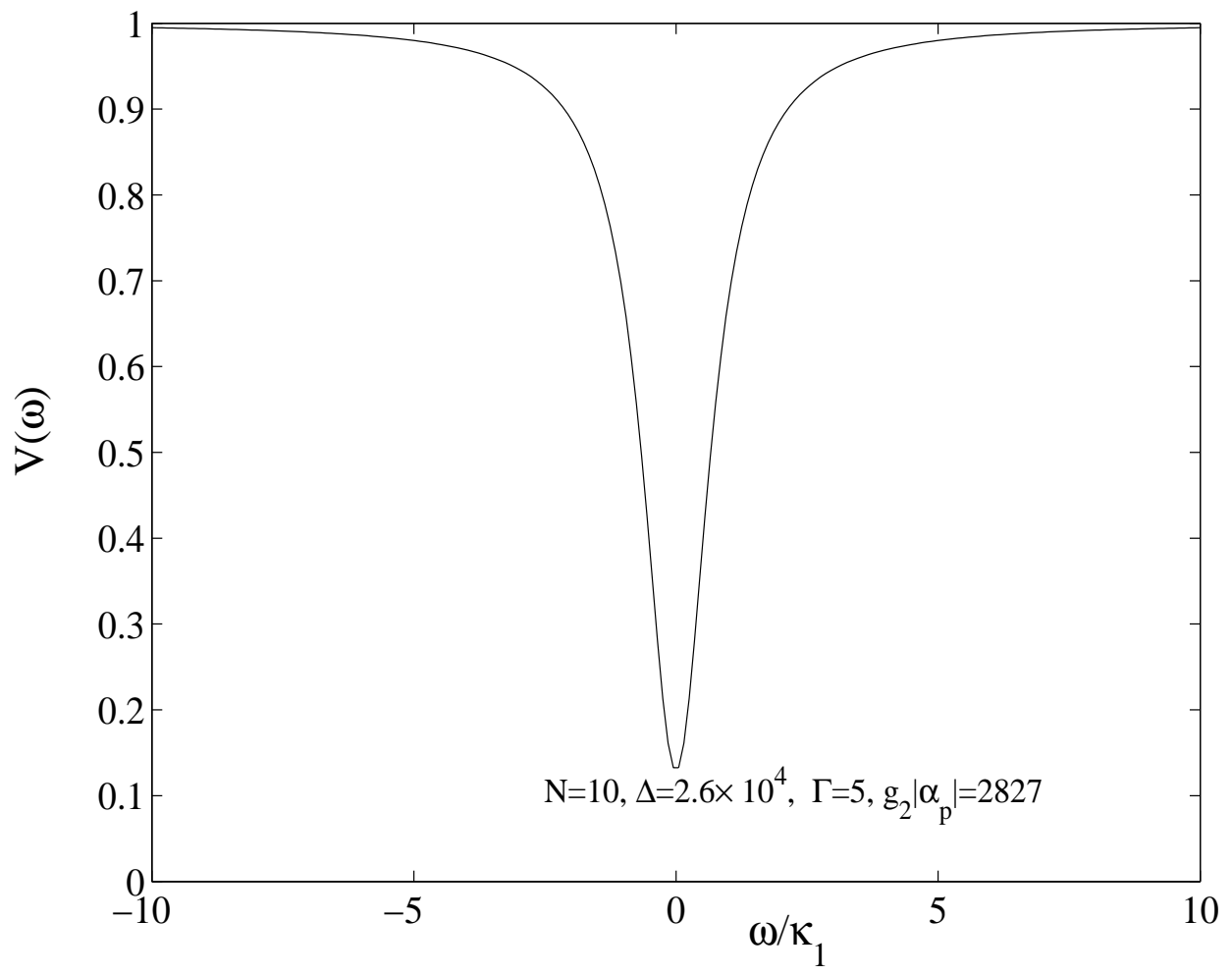

FIG. 2: The quadrature variance $V(\omega)$ for $X_{1}$ is plotted as a function of $\omega$ for $N=10, \Delta=$ $2.6 \times 10^{4}, \Gamma=5, g_{2}\left|\alpha_{p}\right|=2827$ in units of $\kappa_{1}$. The maximum squeezing is obtained at $\omega=0$. 


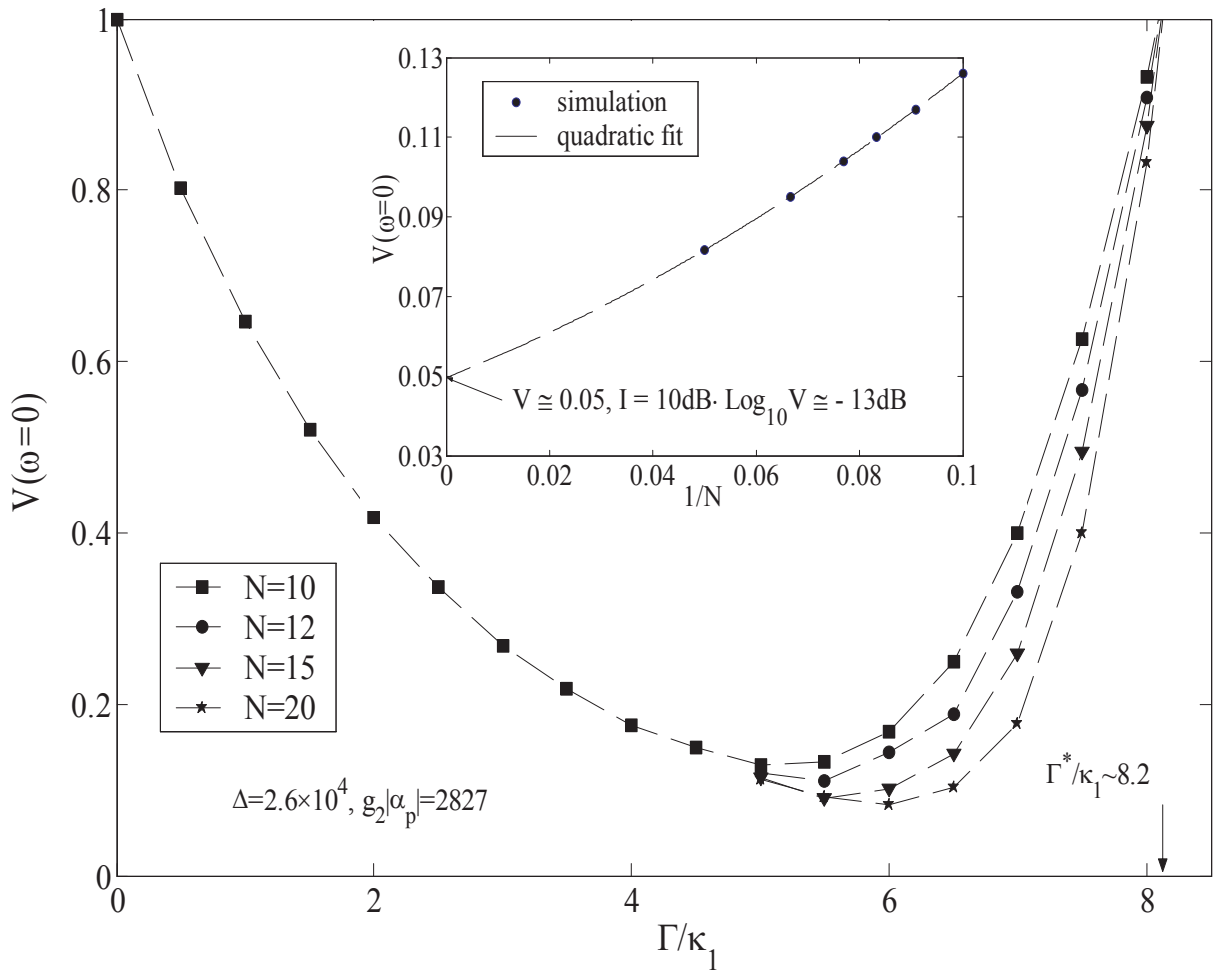

FIG. 3: The quadrature variance $V(\omega=0)$ for $X_{1}$ is plotted as a function of $\Gamma$ for several different values of $N=10,12,15,20$. The minimum value of $V(\omega=0)$ decreases with the increase of the maximum photon number $N$. The critical value of $\Gamma$ is about 8.2 in units of $\kappa_{1}$. In the inset, the maximum output squeezing for several values of $N$ is plotted with respect to $1 / N$. By extrapolation to $N=\infty$, we obtain the $V_{\min }(\omega=0)=0.05$, that is, $-13 \mathrm{~dB}$. 


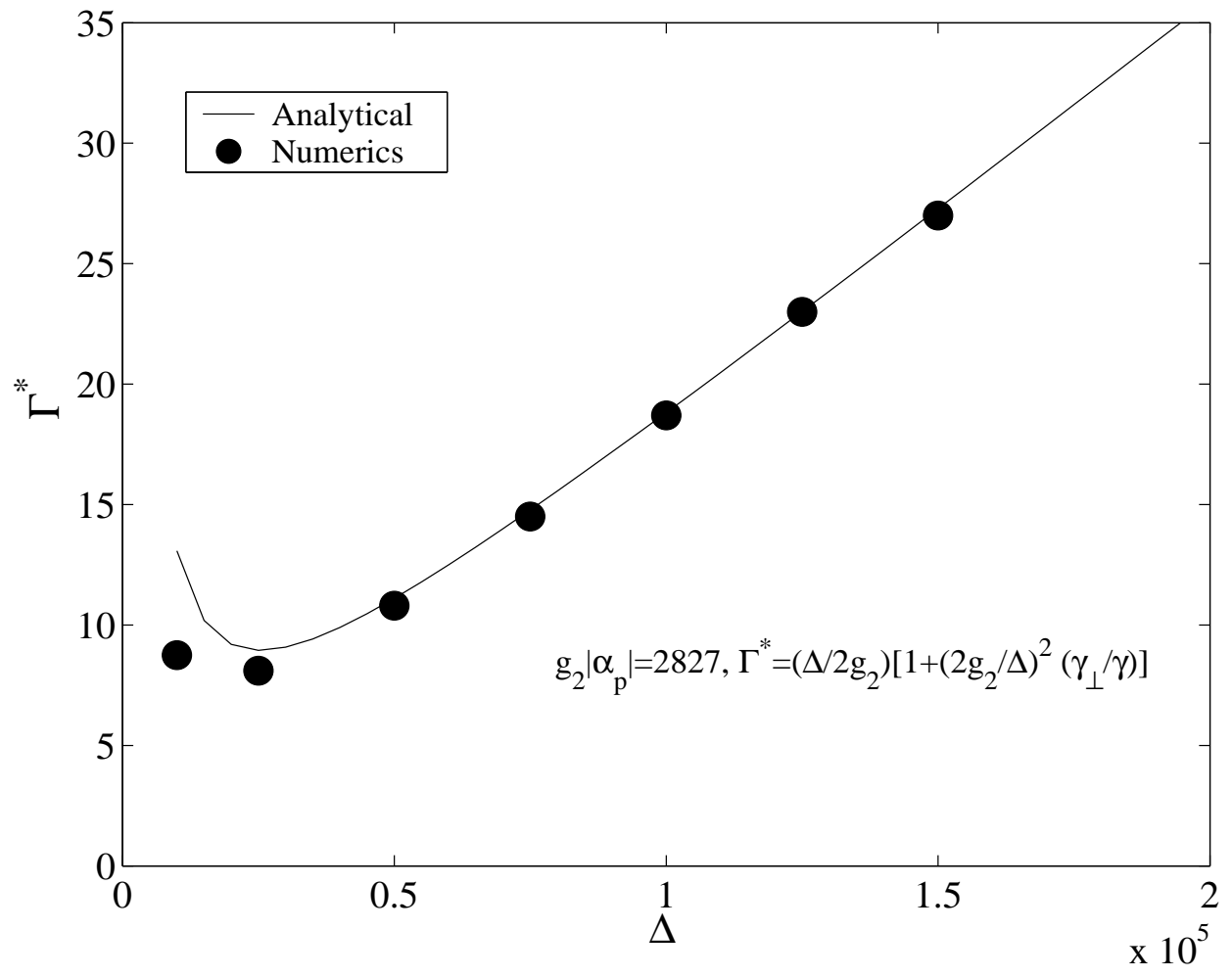

FIG. 4: The critical value $\Gamma^{*}$ is plotted as a function of $\Delta$. The dotted line represents the result from the analytical formula and the filled circles from the numerical simulation. Here $\Gamma^{*}$ and $\Delta$ are in units of $\kappa_{1}$. 